\title{
Fault Feature Extraction of Rolling Bearing Based on LFK
}

\author{
Yu He ${ }^{1, a}$, Li Hongru ${ }^{1, b}$ and Sun Jian ${ }^{1, c}$ \\ ${ }^{1}$ Mechanical Engineering College, Shijiazhuang 050003 , China \\ a13832329446@163.com, ' lihr168@sohu.com, 'c978373767@qq.com
}

Keywords: LCD, cross correlation coefficient, Fast Kurtogram, multi-scale entropy

\begin{abstract}
Based on multiple embedding theory, traditional multi-scale entropy is optimized by local characteristic-scale decomposition (LCD) and fast kurtogram (FK) which can be called LFK for short. In the improved method, the vibration signal of rolling bearing is decomposed by LCD and the two component signals whose cross correlation coefficient with the original signal is bigger than others are selected. FK is applied for filtering the reserved component signal and highlighting the fault feature. Multivariate multi-scale entropy is extracted from the processed signal to characterize the degradation state of rolling bearings. Compared with multi-scale entropy of the original signal, multivariate multi-scale entropy has a better performance.
\end{abstract}

\section{Introduction}

Degradation feature extraction is the key to identify the health status of rolling bearings. However, the rolling bearing vibration signal's nonlinear and nonstationary characteristics lead that the traditional time and frequency domain indexes such as peak to peak cannot clearly describe different degradation states. In contrast, the information entropy theory measures the effective information of the signal from the complexity of the signal and has stronger processing ability for nonlinear signal. The information entropy, such as approximate entropy, sample entropy, multi-scale entropy and so on, is widely used in the field of mechanical fault diagnosis and prediction. By coarse grain, multi-scale entropy (MSE) proposed by Coasta et al[1] ensures that information sequence can be analyzed from the sequence of the whole and the details. However, the vibration signal of the rolling bearing is often affected by the noise and other disturbances, and the complexity of the sequence does not change monotonically with the evolution of the degraded state, and it is accompanied by a strong instability. Therefore, it is necessary to process the signal.

At present, the application of signal denoising methods in engineering applications mainly includes wavelet threshold denoising, empirical mode decomposition (EMD), local mean decomposition (LMD) and local characteristic scale decomposition (LCD). The key problem of wavelet threshold denoising is that it is difficult to accurately select threshold. However, EMD and LMD have defects in theory, such as the mode mixing of EMD [2] and the end effect of LMD, which leads to the lack of the ability of signal decomposition and the poor effect of noise reduction. In contrast, LCD has more advantages in overcoming the endpoint effect, envelope error and decomposition time[3]. In view of the actual complex vibration signal, the traditional filter noise reduction method is difficult to determine the parameters of the filter, the signal type requirements are high, so compared to the previous several methods have certain limitations. Fast Kurtosis Algorithm to kurtosis maximization criterion of band pass filter parameter selection, have a certain effect on the treatment of weak fault signal[4].

Based on the above analysis, a multi scale entropy feature extraction method based on LFK (LCD and FK) is proposed for the processing and analysis of vibration signals of rolling bearings. Multivariate multi-scale entropy is extracted from the signal after LFK processing to distinguish different degradation states of rolling bearing.

\section{The calculation of TMSE}

Assuming that the rolling bearing vibration signal is made up of different ISC components which are unrelated, the LCD method can decompose the vibration signal into $\mathrm{n}$ ISC components and a 
monotonic function[5].

Different ISC components contain different information: some are mainly made up of noise contribution, while others comprise fault feature information. So in order to eliminate the noise component, $m$ orders of ISC components which are closed to fault feature are chosen to reconstruct the new signal .

Cross correlation criterion is used to screen single component signals. By calculating correlation between each ISC component and the original signal, n correlation coefficients can be obtained. The inter relationship of white noise signal and the original signal is zero, but due to LCD, the components don't have clear characteristics of white noise. So the relationship between the noise components and the original signal is not zero, but is relatively small. Based on this, the components whose correlation coefficient is bigger are selected for further analysis.

The basic principle of the FK algorithm is building a set of bands of different $1 / 3$ of binary timberline pass filter in order to achieve sub bands in the kurtosis calculation. Finally, by comparison of the SK of the sub bands, the sub band corresponding to the maximal SK is taken as the optimized demodulation frequency band. Detailed steps are shown in reference [6].

Multiple embedding theory is introduced in multivariate multi-scale based on MSE and the multiple component signals can be analyzed simultaneously. Considering calculation time and complexity, according to the correlation coefficient maximum criterion, two single component signals whose length is $\mathrm{n}$ are selected to do coarse graining and two element embedding reconstruction. Composite delay vector and threshold are set and when the similar capacity limit is $\mathrm{r}$, the conditional probabilities of two composite delay vectors whose embedding vector is $\mathrm{m}$ and $\mathrm{m}+1$ are computed. Then the two-element multi-scale entropy (TMSE) can be expressed as the natural logarithm of the ratio of the two conditional probabilities[7]:

$$
\operatorname{TMSE}(M, \lambda, r, N)=\ln \left[\frac{P^{m}(r)}{P^{m+1}(r)}\right]
$$

Where $M$ is embedding vector and $\lambda$ is delay vector.

\section{Simulated Analysis}

In order to verify the noise reduction effect of the proposed method, the fault signal is set up as follows:

$$
x(t)=0.1 t^{2} \times e^{-c \times \bmod \left(t, T_{0}\right)} \sin \left(2 \pi f_{n} t\right)+n(t)
$$

The coefficient $\mathrm{c}=60$, and $\bmod$ is a residual function. $T_{0}=0.1 \mathrm{~s}, f_{n}=270 \mathrm{~Hz}$. And $n(t)$ is superposed Gauss white noise whose SNR is $-5 \mathrm{~dB}$. The sampling frequency is set as $2048 \mathrm{~Hz}$ and the number of sampling points is 20480. The simulation signal is shown in Figure 1. The shock characteristics have been completely submerged in the noise.

Through the LCD, the simulation signal is decomposed into 10 single component signal and the correlation coefficients of each component signal and the original signal are shown in Figure 2.

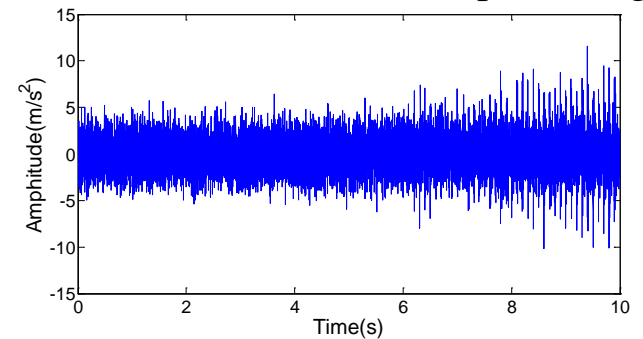

Fig.1 Time domain wave of simulation signal

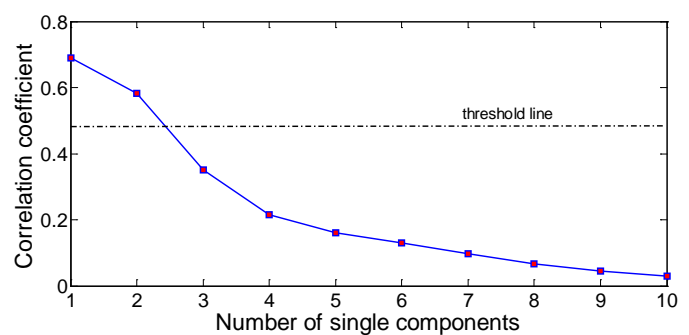

Fig.2 Coefficients of different components

The FK algorithm takes the first two components whose coefficient is relatively large for further analysis. The result is shown in Figure 3, the filter parameters are chosen according to the principle of maximum kurtosis .The center frequency is $f_{c}=128 \mathrm{~Hz}$ and the bandwidth is 
$B w_{0}=256 \mathrm{~Hz}$.And then, the filtered signal's envelope is analyzed in Fig.4. We can see clearly the impacts at the characteristic frequency and its first seven orders. In the whole frequency range, the noise can be effectively suppressed and it doesn't affect the extraction of feature frequency.

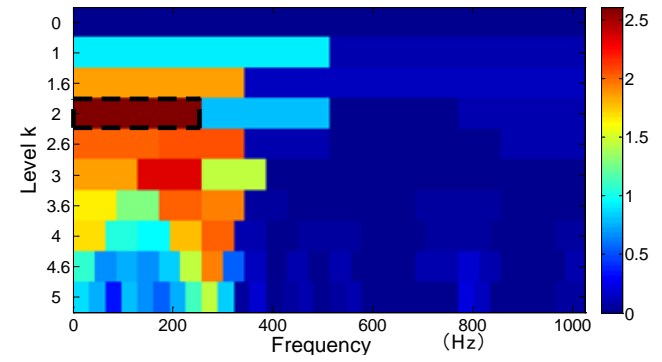

Fig.3 Fast Kurtogram of two-order signal

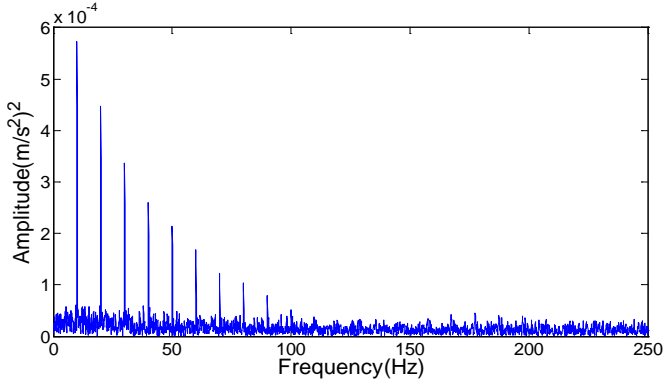

Fig.4 Envelope analysis of two-order signal

Two single component signals preprocessed by LFK algorithm are taken as two single channel signal source. Based on this, multivariate multiscale entropy is calculated and the feature of TMSE is extracted finally.Here are curves of TMSE in different scales in Figure 5. With the deepening of the fault degree, TMSE performs monotonically increasing trend. The greater the scale factor is, the more smooth the curve is and the stronger the ability to characterize degradation state is In contrast, MSE of the original signal is greatly affected by the noise, which does not have the monotonic property that is shown in Figure 6 and the ability to characterize the degradation state is relatively poor.

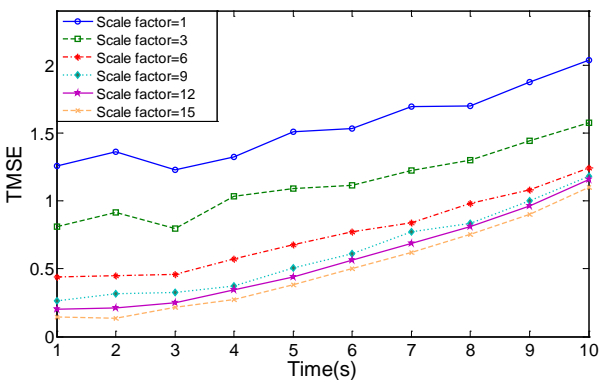

Fig.5 Feature of TMSE

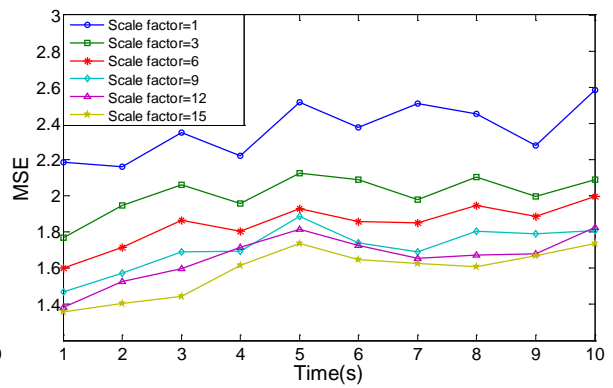

Fig.6 Feature of MSE

\section{Instance signal verification}

The inner ring fault signal data from the Case Western Reserve University Electrical Engineering Laboratory is analyzed in this part[8]. The test object is SKF6205-2RS deep groove ball bearing and the test board is shown in Figure 7. The local fault of different degrees is set up by means of spark machining. The diameters of fault are set as $0 \mathrm{~mm}$ (normal), $0.18 \mathrm{~mm}, 0.36 \mathrm{~mm}, 0.54 \mathrm{~mm}$ and $0.72 \mathrm{~mm}$ which represent 5 different degrees of fault The sampling frequency is set as $12 \mathrm{kHz}$ and the number of sampling points is 24000 . The motor speed is $1797 \mathrm{r} / \mathrm{min}$. The time domain wave of normal state is shown in Figure 8.

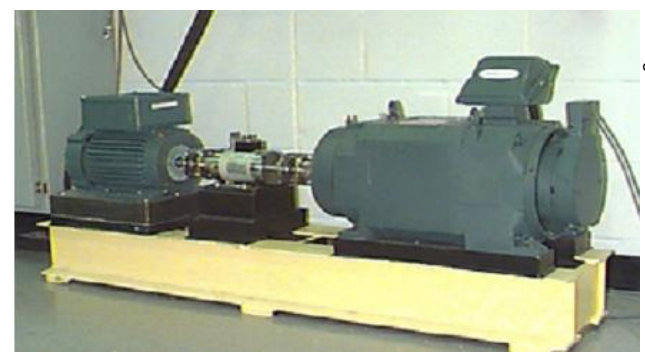

Fig.7 Test board of rolling bearing

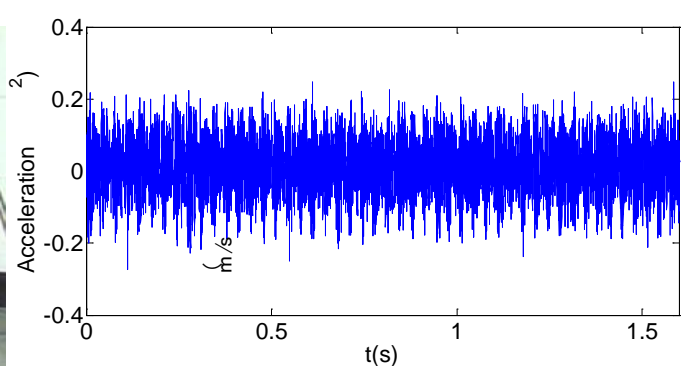

Fig.8 The time domain wave of normal state

After LFK preprocessing, the signals of different fault degrees are analyzed by TMSE firstly. The embedding vector is set as $M=2$ and the delay vector is taken as $\lambda=1$. The similar capacity limit is $r=0.15 \times$ std where std is the standard deviation of the normalized sequence. 24000 points of each signal sequence is divided into 10 groups which contain 2400 points each. The TMSE 
waveforms of different degradation states are shown in Fig.9. Five different degradation states are well distinguished while each fault state's TMSEs are relatively stable. However, without LFK preprocessing, the original signal is analyzed by MSE which is shown in Fig.10. There is a clear overlap between different fault states and MSEs of the same degradation state float largely and randomly. The result illustrates that LFK algorithm has a good performance to reduce the impact of random interference factors such as noise and make the sequence more stable. In comparison, TMSE is more suitable for fault state identification.

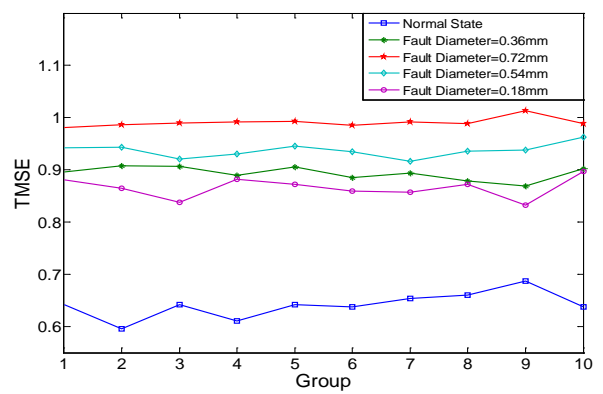

Fig.9 TMSE waveforms of different states

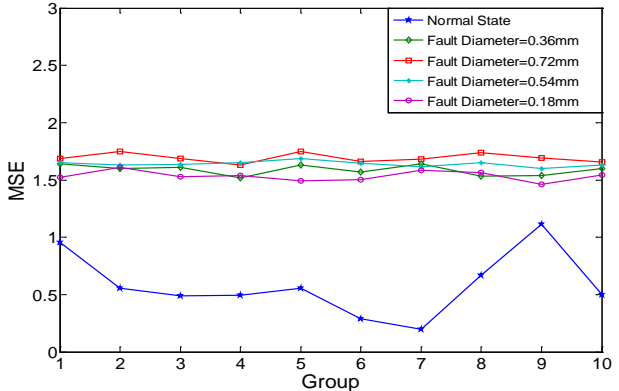

Fig.10 MSE waveforms of different states

\section{Summary}

In order to extract perfect features which can characterize different fault degrees from the complexity noisy environment, a fault feature extraction method based on LFK is proposed in this paper. By means of LFK, the impact of noise components can be eliminated and the characteristic impact can be highlighted. The two remained single component signals contain enough fault information to generate degradation features with relatively small amount of computation. Compared with MSE, TMSE which is extracted from the two processed components has a better ability to characterize the degradation states of rolling bearings.

\section{Acknowledgments}

This project is supported by National Natural Science Foundation of China (Grant No.51541506).

\section{References}

[1] Li Li,Zhu Yongli, Songyaqi.Feature Research of Vibration Signal of Power Transformer Using Multiscale Entropy[J].Journal of Vibration,Measurement\&Diagnosis, 2015,35(4):757-761.

[2] Marcelo A. Colominas, Gastón Schlotthauer, María E. Torres. Improved complete ensemble EMD: A suitable tool for biomedical signal processing. Biomedical Signal Processing and Control, 2014,14 (3): 19-29.

[3] CHENG Jun-sheng, YANG Yi, YANG Yu. Local characteristic-scale decomposition method and its application to gear fault diagnosis. Journal of Mechanical Engineering,2012,48(9):64-71.

[4] PENG Chang, Bo Lin, XIE Xiaoliang. Fault diagnosis method of rolling element bearings based on EEMD, measure-factor and fast kurtogram. Journal of Vibration and Shock, 2012,31(20):143-146.

[5] YANG Yu, ZENG Ming, CHENG Junsheng. Research on local characteristic-scale decomposition and its capacities. Journal of Vibration Engineering, 2012,25(5):602-608.

[6] DAI Shichao, GUO Yu, WU Xing, et al. Improvement on fast kurtogram algorithm based on sub-frequency-band spectral kurtosis average. Journal of Vibration and Shock, 2015,34(7):98-102.

[7] Xu Yonghong, Cui Jie, Hong Wenxue, et al. Automatic Classification of Epileptic Electroencephalogram Signal Based on Improved Multivariate Multiscale Entropy[J]. Journal of 
Biomedical Engineering,2015,32(2):256-262.

[8] Information on http://www.eecs.cwru.edu/laboratory/bearing 